le portiQue $\begin{array}{ll}\text { Le Portique } \\ \text { Revue de philosophie et de sciences humaines }\end{array}$

3| 1999

Technique et esthétique

\title{
Peinture et cinéma
}

(une esquisse)

Walter Benjamin

\section{(2) OpenEdition}

Journals

Édition électronique

URL : http://journals.openedition.org/leportique/311

DOI : 10.4000/leportique.311

ISSN : $1777-5280$

Éditeur

Association "Les Amis du Portique"

Édition imprimée

Date de publication : 1 janvier 1999

ISSN : 1283-8594

Référence électronique

Walter Benjamin, «Peinture et cinéma », Le Portique [En ligne], 3 | 1999, mis en ligne le 15 mars 2005, consulté le 25 mars 2021. URL : http://journals.openedition.org/leportique/311 ; DOI : https://doi.org/ 10.4000/leportique.311

Ce document a été généré automatiquement le 25 mars 2021.

Tous droits réservés 


\title{
Peinture et cinéma
}

\author{
(une esquisse)
}

Walter Benjamin

1 Jamais, pas même à l'époque de son efflorescence, la peinture n'a été directement reçue par les masses. Toujours sa réception est passée par certaines médiations sociales où elle était résolument malmenée. « Mais jetons, dit Le Corbusier, un regard sur l'histoire. L'homme simple - je parle avant tout du paysan dont les obligations impliquaient qu'il aille de temps en temps en ville ou qu'il se rende au château - n'avait ni les présupposés culturels, ni la liberté, ni l'esprit nécessaire pour comprendre les divines proportions de l'œuvre d'art. En revanche, il y trouvait une harmonie à sa convenance, et il lui suffisait d'y saisir au passage un certain nombre d'éléments superficiels; il utilisait ensuite ces éléments selon des critères tout à fait personnels, les organisait en fonction de son propre arbitraire, ne respectait pas leurs proportions, les déformaient sans tenir compte de leurs propriétés les plus essentielles, puis, ainsi chargé d'un miel tout différent, il rentrait chez lui. Après un tel massacre, il se mettait à créer son œuvre en obéissant à des harmonies toutes personnelles, c'est ainsi qu'apparurent les merveilleuses œuvres d'art du folklore.»

2 En jetant un regard rétrospectif sur la situation antérieure, Le Corbusier rejette cette vague « intelligibilité générale » des tableaux par laquelle on a cherché à disqualifier la peinture des années vingt. Le Corbusier rappelle les formes qui ont toujours présidé à la réception de la peinture précisément : c'étaient des formes hiérarchiques. La meilleure idée de son schéma nous est fournie par la mode qui à chaque fois qu'elle est reçue par des couches plus importantes subit des changements : plus les couches sont larges plus les changements sont grands. Le Corbusier constate à juste titre que le fondement d'une telle conception hiérarchique s'est perdue pour la peinture. Il ne va, au demeurant, pas plus loin. Il ne soulève pas la question de ce que signifie pour la peinture d'être (comme le cinéma) soumis à l'exigence d'entrer en contact direct avec la grande masse. Or c'est cette exigence, non sans rapport avec les techniques modernes de reproduction, qui s'est effectivement imposée. Une tentative précédente d'y satisfaire fut les expositions artistiques. On ne saurait guère aujourd'hui contester 
que la procédure a échoué et que la peinture se soustrait de ce point de vue aussi à la confrontation directe avec la masse a.

La réception de la peinture mérite d'être considérée de plus près sous un autre rapport.

Un élément important vient s'ajouter : la réception se modifie. Parler de la réception de la peinture signifie en premier lieu rendre compte de son rapport à l'architecture. Forcément, on ne rencontre pas de tableaux dans la rue. Même leur exposition dans des Salons représente une exception, qui ne va absolument pas sans être problématique, à son mode de conservation naturel dans la pièce d'habitation. Il y a cent ans Balzac ${ }^{b} \mathrm{a}$ été l'un des premiers à mentionner le fait, lourd de conséquence, que les logements deviennent de plus en plus petits. Ce que cela veut dire pour la peinture, un regard sur la Renaissance le montre. Il n'est sûrement pas exagéré d'expliquer que la signification de la perspective dans cette peinture ne peut être dissociée de la signification de cette architecture pour cette peinture. Les peintres de la Renaissance ont mis pour la première fois des intérieurs dans leurs tableaux où les personnages portraiturés disposent d'une aire de jeu: des pièces où bien agir pouvait passer pour une tâche de ceux qui les habitent. La peinture, qui mettait ses créations à la disposition du décor de la pièce d'habitation, leur a été d'une grande aide dans l'accomplissement de cette tâche. Elle s'est solidarisée avec les habitants en ouvrant inlassablement dans ses tableaux des perspectives prenant la forme d'un intérieur (qui, en tout état de cause, n'avait pas encore de caractère intime). Si l'on songe à cela, on comprend que Le Corbusier ait pu déclarer à Venise : "Cette production picturale énorme, qui passe complètement de nos jours à côté de l'architecture, me fait peur. ${ }^{\mathrm{c}}$ Dans la grande majorité des petits logements d'aujourd'hui, quand ce ne sont pas des taudis - les gens n'ont plus suffisamment d'aire de jeu pour pouvoir exploiter le modèle des figures empruntée au trésor des postures de la peinture (pour ne rien dire des figures de majesté qu'ont connues la Renaissance ou le baroque). Des nombreux éléments qui déterminent la réception, une telle possibilité d'exploitation n'en constitue assurément qu'une. Toujours est-il qu'elle ne saurait faire défaut sur toute la ligne et pour une durée indéfiniment longue sans porter préjudice à la réception de la peinture. Ce qui est décisif, c'est non pas le rapport plus ou moins mécanique entre peinture et architecture, ni la plus ou moins grande place sur le mur, ni le plus ou moins grand recul par rapport au tableau que permet la pièce, mais c'est le rapport entre l'habitant et sa pièce d'habitation. C'est de lui que dépend en premier lieu que le tableau y ait une place. C'est inclure un pronostic sur la réception de la peinture que de constater comme Le Corbusier que les logements dans les villes du monde sont indignes de l'homme; qu'on les a sacrifiés au profit.

On peut hésiter quant au facteur qui pèse le plus lourd: celui qui est présent dans la grande majorité des situations de logement d'aujourd'hui ou bien celui qui constitue l'exception. On ne doit, en effet, pas oublier que dans les maisons susceptibles d'être fabriquées pour leurs propriétaires selon le dernier standard de l'architecture, la place du tableau n'est pas moins problématique que dans les autres. Les bâtiments des nouveaux architectes, de l'école hollandaise de [un mot effacé], de l'école allemande de Hannes Meyer, de l'école française de [un mot effacé] remplacent les cloisons statiques, planes et opaques de la pièce d'habitation par des cloisons coulissantes, curvilignes et transparentes. Lambotte, l'ancien directeur de l'administration des arts en Belgique, pense à cela quand il dit mélancoliquement : «Le nouveau style n'a plus besoin du tableau. Faut-il s'en féliciter?" S'agirait-il seulement d'une question de style, on 
n'aurait pas à prendre la chose au sérieux. En vérité il s'agit de la modification du sentiment spatial humain dont le nouvel habitat, la "machine à habiter ", n'est qu'un symptôme. On peut en déceler un autre chez l'homme sans maison, libre de ses mouvements ou plutôt libéré des lois de la pesanteur.

Durant des millénaires, la verticale a été l'axe d'où l'homme a contemplé le monde. Même les tableaux l'abordaient debout, à la verticale d. Depuis que l'avion est en usage, le monopole de la verticale est entamé. Une percée d'une importance incontestable a été réalisée. Wallon, un matérialiste dialectique, est un des premiers à avoir pris en compte ce phénomène. Wallon dit: "L'usage de l'avion a inéluctablement modifié notre manière de voir. Depuis, nous connaissons la perspective aérienne, les raccourcis et toutes sortes d'angles de vue insolites. Avec l'usage de l'avion la verticale perd sa fixité inébranlable. Tout ce qui va et vient à la surface de la terre ne connaît pas d'autres déplacements qu'en avant ou en arrière, à droite et à gauche et les différentes combinaisons de ces mouvements. L'avion ajoute une troisième dimension, il combine avec elles des déplacements à la verticale, c'est-à-dire en direction de la pesanteur. » Wallon insiste sur l'intensité particulière que prennent du fait de la rapidité des mouvements qui leur sont associés les nouvelles expériences que fait le corps dans l'espace et il ajoute : « Il apparaît ainsi incontestable que les nouvelles inventions de la technique ont pour conséquence de nouvelles réactions de notre appareil sensoriel [...] N'est-il pas nécessaire que nos nouveaux automatismes aient des répercussions jusque sur notre système musculaire, notre sensibilité et, pour finir, notre intelligence ?» ([La Querelle du réalisme, Paris, 1936] p. 145-147) Il n'est pas nécessaire de remarquer que l'exposé de Wallon vaut aussi pour celui qui ne s'est jamais assis dans un avion. Car cela ne l'empêche pas de frayer, par empathie, la voie à ces automatismes. En effet il est amené de manière absolument continuelle à cette empathie pour l'appareil. Et ce par le cinéma et le journal illustré. La caméra est capable de s'adapter aux nouvelles conditions de la vue mieux que l'œil humain. Dans ses évolutions, le pilote a exclusivement en vue la machine. Il est rare que la capacité d'assimilation du passager de l'avion reste absolument intacte lors de forts déplacements à la verticale. Précisément les vues du paysage aérien les plus mouvementées et les plus excitantes s'offrent d'abord à la caméra qui les communiquent ensuite à l'œil. (C'est ce qui fait l'originalité particulière de ces vues.)

7 L'expérience qui se fait ainsi jour peut être résumée ainsi : l'efficience spécifique de l'appareillage photographique fait ses preuves avec certaines tâches qui échappent à la peinture. Cette proposition peut être appliquée non seulement à la sphère technique mais aussi à la sphère économique et elle prend ainsi une portée particulière. Elle est au demeurant tout à fait apte à donner une vue d'ensemble sur la crise de la peinture qui est l'objet de nos congrès.

En effet, le caractère économique de la photographie n'est pas épuisé avec le fait d'être une marchandise. Elle partage ce caractère avec tous les produits de notre société. (La peinture, elle aussi, y est une marchandise.) La marche triomphale de la photographie repose sur un élément supplémentaire. La photographie n'est pas seulement une marchandise, elle rend aussi certains services à l'économie marchande en général. C'est tangible à partir de Disdéri. Disdéri a été le premier - comme photographe de portraits - qui a consciemment diffusé ses produits comme une marchandise. Mais il est allé plus loin : il a également été le premier qui, par le moyen de la photographie, a intégré dans le processus de circulation d'autres biens qui échappaient plus ou moins à celui-ci. 
Ainsi, en premier lieu, les œuvres d'art. Disdéri avait eu l'idée intelligente de se faire donner par l'État un monopole sur la reproduction des œuvres d'art rassemblées au Louvre. Depuis, la photographie a rendu commercialisables des secteurs toujours plus nombreux du champ de perception optique. Indirectement mais de manière décisive la photographie d'amateur a profité à cette évolution. Avec elle, au tournant du siècle, la mode a commencé à intervenir dans l'arrangement de l'image. La photographie se retrouva ainsi en position de faire passer continûment, par le biais de fugitives touches de mode, n'importe quel sujet (et pas seulement des sujets d'agrément ou d'actualité) dans la presse illustrée. Elle a acquis à la circulation des marchandises des objets qui autrefois n'y avaient pour ainsi dire jamais eu leur place ${ }^{1}$.

9 La rapide usure de la photographie est naturellement inséparable de cette fonction. Personne n'aurait eu l'impertinence de l'envisager pour le tableau; la durée relativement longue de sa fabrication la rendrait insupportable. Mais si l'on veut s'imaginer quel pourrait être le mode d'existence de la peinture dans de telles conditions, il n'est pas besoin d'aller bien loin. La vie quotidienne donne souvent la preuve par un exemple de l'histoire du monde. Le seul lieu qui ait encore à passer massivement des commandes, c'est le cinéma. En particulier les petites salles de cinéma qui évitent les coûteuses enseignes lumineuses donnent une idée de leur programme par des images peintes. Ces affiches équivalent à une exécution historique du tableau. Celui-ci est traîné dans la rue; on lui arrache son cadre; sa durée de vie est de huit jours ; pour quiconque sort du cinéma son sujet est sans valeur.

10 Si l'observation de la photographie nous apprend quelque chose, c'est que les images, en dehors de l'utilité qu'elles pouvaient avoir dans les époques passées, peuvent correspondre à certaines finalités qui, parce que déterminées par les besoins d'une société productrice de marchandises, n'existaient pas autrefois. Que la photographie réponde mieux que la peinture à ces nouvelles finalités, voilà ce que la technique de la réclame a mis à profit avec le plus de cohérence. On ne peut pas reprocher à la technique de la réclame de négliger l'aspect esthétique de ses productions. Il y a quantité d'images de réclame qui se gravent dans la mémoire parce qu'elles sont séduisantes. Que cette séduction repose non pas sur leurs éléments artistiques autonomes mais sur la manière dont ceux-ci sont liés aux éléments informatifs modifie la qualité de cette séduction mais ne la diminue pas. Ces éléments informatifs sont pour la plupart du temps présents dans l'image de la marchandise elle-même. Et de plus on s'en remet pour la restitution en image de la marchandise à la photographie e. Les firmes d'automobiles, de cigarettes, de textiles ont peu à peu éduqué le peuple à chercher certaines qualités marchandes dans l'image. Elles ont placé la photographie à la place de l'échantillon de marchandise. Cette place, la peinture ne pouvait pas l'occuper. Voilà qui livre accès à un autre phénomène important: le rythme de développement de la technique de production a fait qu'il est devenu de plus en plus important de pouvoir prendre des échantillons de cette marchandise que représente la force de travail humaine. Depuis l'invention du cinéma, c'est devenu possible sans aucune difficulté. Nous possédons des enregistrements cinématographiques de toute une série de tests d'aptitudes. Mais cette constellation des tests d'aptitude existe désormais sous mille formes. Plus la lutte pour l'existence se fait vive, plus les situations et les modes de comportement qu'on reconnaissait appartenir à la vie privée deviennent pertinents. On ne saurait dénier au test d'aptitude, aussi dénué d'objectivité soit-il, comme dans les États fascistes, qu'il soit totalitaire et s'étende à l'ensemble des faits de la vie de la personne testée (et même le cas échéant à la lignée de ses ancêtres). 
Il peut au demeurant - comme le montre l'Union soviétique - avoir un rayon très large sans pour autant perdre en objectivité. En effet l'étendue des faits de l'existence humaine susceptibles d'être soumis à des tests (par suite de la lutte des intérêts plus vive et des méthodes de travail plus affinées) est aujourd'hui plus vaste que jamais. Nous rencontrons ici une prestation sociale décisive du cinéma. Il a fait entrer l'homme, dans toute l'étendue de son existence visible, à l'intérieur d'un laboratoire. Son importance repose en grande partie sur son aptitude à fixer des réactions humaines dans n'importe quelle constellation - des constellations qui apparaissent au public de cinéma pour ainsi dire comme un test complémentaire même s'il est la plupart du temps moral et rarement techniquement professionnel ${ }^{\mathrm{f}}$. Il s'ensuit que ce public peut difficilement être celui de la peinture. Celle-ci peut seulement prétendre à un vaste public tant que l'homme visible peut être représenté par elle dans des situations et des contextes où ses contemporains lui témoignent un intérêt particulier. Il n'est pas dit que sa fonction dans le processus de travail ait toujours été celle-là. Mais il est tout de même caractéristique que le processus de travail se soit très bien laissé dépeindre durant des siècles. Le développement technique du dernier siècle a beaucoup limité cette possibilité. Menzel et Liebermann ont encore pu peindre au siècle dernier des secteurs de l'activité industrielle - ultérieurement c'est devenu plus difficile. Car, d'abord, la capacité de la peinture à pouvoir fixer un processus dans son déroulement est très limitée $\mathrm{g}$. De plus la peinture n'est pas en mesure de fixer des processus où l'exactitude de la procédure est décisive.

11 Nous ne possédons pas d'exemple plus concluant que le film pour montrer à quel point sont étroitement imbriqués ces deux éléments : l'utilité d'une nouvelle technique pour des besoins économiques qui se sont modifiés, l'utilité d'une nouvelle manière de voir pour des besoins esthétiques qui sont devenus différents. Le réalisme socialiste n'a aucune raison de dédaigner ces corrélations. Si elles ont été peu mises en valeur au cours des débats parisiens - ou même, comme nous avons entendu Aragon le dire, sabotées par une série de participants, cela peut tenir à la nature d'un tel débat public. La conséquence préjudiciable en a été que la majorité des participants n'espérait trouver le salut que dans de nouveaux sujets. La réaction à ce malentendu va de soi et Gaillard lui a donné sa formulation. «Pour que les drames sociaux, dit-il, aient une place dans le sujet de mes tableaux, il faudrait que je puisse en être visuellement impressionné. D'un autre côté je suis d'avis que les combats sociaux exercent [...] une influence sur ma vie et laissent ainsi leur marque dans mon œuvre. ${ }^{2}$ De telles réflexions font bien voir qu'il n'est pas pertinent de discuter du réalisme socialiste sous le seul rapport du sujet. Il signifie en outre pour la peinture ceci : l'évaluation réaliste de ses données sociales. Beaucoup de choses dépendent d'une juste connaissance de ses points d'intervention. Peut-être ceux-ci ne peuvent-ils pas s'observer aussi nettement dans un pays qui a encore des libertés démocratiques ${ }^{\mathrm{h}}$ que dans les pays où le fascisme tient le gouvernail. Là-bas la peinture est vivante dans l'exacte mesure où elle dispute la vie au fascisme. Elle n'est pas l'œuvre des anciens futuristes qui ont fait leur entrée solennelle à l'Académie royale italienne et dont beaucoup pourraient rejoindre Dufy qui a déclaré que, s'il était un Allemand et devait célébrer le triomphe de Hitler, il ferait comme certains peintres du Moyen Âge qui ont traité des thèmes religieux sans être pour autant croyants ${ }^{\mathrm{i}}$. Il $\mathrm{y}$ a en Allemagne des peintres qui procèdent autrement. Il leur est interdit de peindre, d'exposer publiquement leurs tableaux. La police organise dans leur logements des razzias d'œuvres fraîchement peintes. Ces artistes qui, sans moyens de subsistance, sont assis la nuit devant leurs paysages calcinés piqués de drapeaux, 
leur portraits de dignitaires camouflés en figures animales savent ce qu'est le réalisme socialiste. Le savent tout autant ceux dont on a interrompu l'activité à cause de la manière dont ils peignaient. Ils seront très loin de voir dans le réalisme socialiste une simple affaire de sujet. Ils seront tout aussi éloignés de la certitude que la peinture a atteint un niveau qui satisfait aux besoins du réalisme socialiste et qu'il lui reste seulement et simplement à amener les masses à jouir de cette peinture. Léger se trompe. À cette tâche politique la peinture est bien destinée à prêter son concours. "Parmi les œuvres les plus importantes en peinture [...]», écrit René Crevel, récemment décédé, «il a toujours fallu compter celles qui, du fait même qu'elles constataient une décomposition, requéraient contre ses responsables. De Grünewald à Dali, du Christ pourri à l'âne pourri [...] la peinture a toujours su trouver [...] des vérités nouvelles qui n'étaient pas seulement d'ordre pictural. ${ }^{3}$ Sur cette voie, où se situent finalement les œuvres d'un Goya et d'un Daumier, se trouvent également ces peintres allemands mis au ban. Le fascisme leur a montré ce qui est utile dans une image aujourd'hui : cette marque publique ou secrète qui prouve qu'elle provient de l'ennemi. L'exemple classique que nous en avons est celui des caricaturistes dont l'intérêt passionné pour les luttes sociales a pour conséquence que leur savoir politique imprègne tout autant leur perception physionomique que notre savoir sur les rapports de l'espace imprègne nos perceptions quotidiennes (comme l'a prouvé la psychologie de la perception). [La phrase est obscure du fait de l'oubli par Benjamin du sujet du second verbe et le sens restitué ici est seulement probable.] Des maîtres comme Bosch, comme Hogarth, comme Daumier ont indiqué cette voie à la peinture. Le tableau de Delacroix La Barricade a eu un effet politique d'une extrême virulence. Les tableaux plus récents de Dix et de George n'en ont pas eu moins. Ces exemples suffisent à montrer que le sujet social n'est pas décisif. Ce qui est décisif c'est ce qui s'exprime nettement dans le sujet. Il tient à la nature de la situation ouest-européenne que la peinture, précisément là où elle aborde souverainement son objet, ait un effet destructeur, purificateur.

@ S Surkamp Verlag, Francfort-sur-le-Main, tous les droits réservés.

(c) Le Portique, Éditeur et Jean-François Poirier pour la traduction française.

\section{NOTES}

a. Avec la réception massive du matériau-image, c'est devenu un besoin des masses de se regarder soi-même dans les yeux. Ce besoin est satisfait par la presse illustrée et les cinémas. Les peintres qui ont certes senti combien ce nouveau sujet qu'est la masse est 
réceptif à la publicité ne peuvent pas entrer en concurrence avec ces institutions. Mais il est peu de choses qui seraient plus riches d'enseignement pour le siècle dernier que d'exposer les tentatives qui ont été entreprises en ce sens. «La masse comme sujet dans la nouvelle peinture » livrerait un travail très instructif. De même Aragon : « Depuis longtemps déjà on ne peint plus les masses. Mais aujourd'hui les masses font de nouveau leur entrée dans l'art par l'appareil photographique».

b. Dans l'histoire riche en épisodes étranges de l'accueil fait à la photographie, l'accueil que lui a réservé Balzac est le plus singulier. Dans Le Cousin Pons Balzac développe une théorie de la prophétie qui est étayée tout du long par l'analogie avec la photographie. Il faut savoir que Balzac concevait le procédé photographique d'après une théorie qui est très semblable à la théorie épicurienne des eidola (qu'il ne connaissait sans doute pas). Selon elle des réductions fidèles à l'original - de petites images - ne cessent de se détacher des choses. Percevoir ces petites images constitue le privilège de celui qui fait des prophéties, sa reproduction celui de l'appareil photographique. Il serait intéressant d'établir quand Balzac a fait les premières déclarations qui vont dans ce sens. On peut penser qu'il a échafaudé cette théorie pour les besoins du passage dans le roman évoqué et puis qu'il s'en est tenu à elle.

c. Est-il trop audacieux de voir un réflexe à une telle peur dans le cubisme qui a inclus cette construction, qui assignait autrefois au tableau une place dans l'espace, maintenant que cette place a disparu, dans le cadre du tableau lui-même?

d. Il n'est pas sans importance de bien se représenter que cela ne vaut pas ou pas toujours exclusivement pour les arts plastiques.

1.. Cf. Walter Benjamin, Sur l'art et la photographie, prés. et trad. de Christophe Jouanlanne, Paris, Éditions Carré, 1997, p. 85-86.

e. Il n'y a là certes rien d'étonnant si l'on songe que la photographie s'est déjà à l'occasion emparée de l'illustration de textes littéraires ou poétiques. Dernièrement on a édité en France non seulement l'auteur de romans policiers Georges Simenon mais aussi Jean Giono et Paul Valéry avec des illustrations photographiques.

f. Le public est soumis, soit dit en passant, à des tests au cinéma lui-même. Sa capacité à comprendre le film est testée. Nous pouvons très bien admettre que les exigences nouvelles et diverses que le cinéma adresse à l'intelligence des gens contribuent à la demande dont il est l'objet.

g. Même si ses efforts en ce sens sont très liée à une époque. Le peintre Valentine Hugo écrit dans Commune: «Je souhaiterais qu'une femme puisse oser ce que personne n'a encore osé jusqu'à maintenant et qu'elle fixe sa propre vie dans une série de tableaux » Ce qui signifierait oser faire un film avec les moyens de la peinture.

2.. Walter BENJAMIN, op. cit., p. 92-93.

h. Encore ceci : à l'occasion de la grande exposition Cézanne le journal parisien « Choc » se donnait pour tâche d'en finir avec le «bluff » Cézanne qu'un gouvernement de gauche avait promu dans le seul but « de traîner dans la boue les convictions artistiques de son propre peuple et même de tous les autres peuples. » (Cf. Walter BENJAMIN, op. cit.

p. 94, traduction modifiée.)

i. Il est certain que le Pérugin était athée.

3.. Cf. Walter BENJAMIN, op. cit., p. 93-94. 


\section{RÉSUMÉS}

Ces deux textes, le premier long et le second très court, constituent une étape préparatoire au texte intitulé «Peinture et photographie » publié en français dans une traduction de Marc B. de Launay in Walter Benjamin, Sur l'art et la photographie, Deuxième lettre de Paris, 1936, présentation et traduction de Christophe Jouanlanne pour les autres textes, Paris, éditions Carré, 1997. Ce long fragment et ce court fragment, répertoriés au Benjamin-Archiv de Francfort-sur-leMain sous les cotes Ms $372-380$ et Ms $364^{v}$, $380^{v}$, ont été publiés en allemand dans le tome VII de l'édition des Gesammelte Schriften, Suhrkamp, 1989, p. 815-823 et le début du texte, jusqu'à la première citation de Le Corbusier comprise, qui est identique à celui d'un autre fragment, figure dans le tome III des G.S., p. 679. Les citations françaises n'étant pas référencées dans l'édition allemande et comme il était impossible dans le cadre de ce travail, notamment en raison de la fermeture de la Bibliothèque Nationale de France, de partir à la recherche des sources, les citations ont été traduites de l'allemand. Les notes appelées par des lettres sont de Benjamin, les notes appelées par des chiffres sont des responsables de l'édition. 